\title{
Inverse Kinematic Model of Flexure-Based Microsurgical Manipulator
}

\author{
Juan García Avedillo ${ }^{1}$, David Y. Choi ${ }^{2}$, and Cameron N. Riviere ${ }^{2}$ \\ ${ }^{1}$ School of Engineering, University of Valladolid, Valladolid, Spain \\ ${ }^{2}$ The Robotics Institute, Carnegie Mellon University, Pittsburgh, PA, USA
}

\begin{abstract}
This paper presents the development of an inverse kinematic model for a flexure-based micromanipulator using a feedforward neural network. The manipulator is part of an active handheld instrument designed to cancel hand tremor during microsurgery. The manipulator is actuated using three piezoelectric stacks. Preliminary results yield a mean squared error of $5 \%$ of the total stack range of motion, and a maximum error of $14 \%$ of the total range of motion.
\end{abstract}

\section{INTRODUCTION}

Physiological hand tremor is inherent in the movement of surgeons. During vitreoretinal microsurgery, the tremor is exhibited as an approximately sinusoidal involuntary motion with typical amplitude of $50 \mu \mathrm{m}$ p-p or less, and a frequency between 8 and $12 \mathrm{~Hz}$ [1,2]. Recent years have seen several projects intended to reduce the tremor of microsurgeons $[3,4]$. In our laboratory, an active handheld micromanipulator known as Micron is being developed [5].

A new tip manipulator for Micron has been fabricated as a single-piece flexure [6]. The benefits of such an approach include are the elimination of errors in pin and ball joints, including friction, backlash, and other nonlinearities due to machining tolerances. Micron's manipulator is a threedegree-of-freedom (3-dof) parallel mechanism driven by three piezoelectric actuators. Each piezoelectric stack is 5 $\mathrm{mm} \times 5 \mathrm{~mm} \times 18 \mathrm{~mm}$ and can deflect about $15 \mu \mathrm{m}$ at +100 $\mathrm{V}$. The stacks are mounted in the structure in a radially symmetric pattern, as shown in Figure 1. The manipulator incorporates a system of levers in order to accomplish mechanical amplification of the movement of the stacks.

For control of such manipulators, the inverse kinematics are often treated analytically [7]. In the present case, the nature of the flexure-based design, the geometric differences between the three linkages and the relatively low precision of the stereo lithographic fabrication process lead to uncertainty regarding the measurements of the prototype, so that careful calibration of a considerable number of parameters in the analytical model would be needed. In the presence of such uncertainties, it is simpler to resort instead to a black box method such as a neural network [8]. This paper presents preliminary results from such an approach.

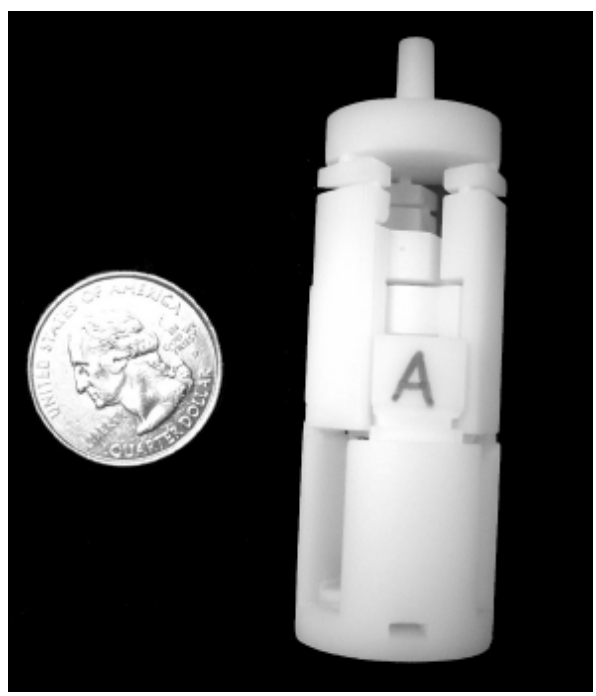

(a)

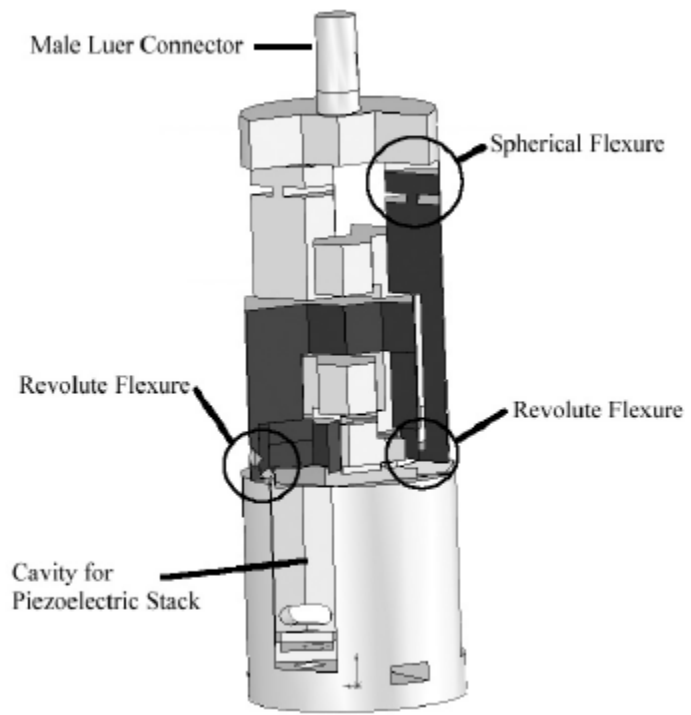

(b)

Fig. 1. (a) Photograph of manipulator, with a U.S. quarter for comparison (b) Diagram showing one lever in a darker shade to display the three flexures. 


\section{METHODS}

Data to train the neural network model were generated by sampling 10,000 points randomly distributed throughout the workspace of the manipulator. Points were generated by a common random number generator function, with the range limited to $0-15$ microns, reflecting the physical limits of the range of motion of the piezoelectric stacks. The cloud of points is depicted in Figure 3.

A custom three-dimensional (3-D) optical tracking system for validation of microsurgical instruments (ASAP, for Apparatus to Sense Accuracy of Position) was used for data collection [5]. Figure 2 shows the setup, with the manipulator affixed to the underside of a small platform near the top of the photograph. It is possible to see a white ball at the manipulator tip. This ball reflects infrared illumination within ASAP, which is then sensed by two position sensitive detectors to provide 3-D tracking of the instrument tip. Tracking data were then transformed from the reference frame of ASAP to that of the manipulator.

A feedforward neural network with a single hidden layer was used to model the inverse kinematics. LevenbergMarquardt backpropagation learning was used to train the network, providing second-order training speed without having to compute the Hessian matrix [9].

The 10000-point data set depicted in Fig. 3 was used to train the network. Separate training and validation data sets were used. The validation data set contained 500 points. The number of hidden nodes was optimized by training networks having from 3 to 9 hidden nodes and choosing the best-performing network.

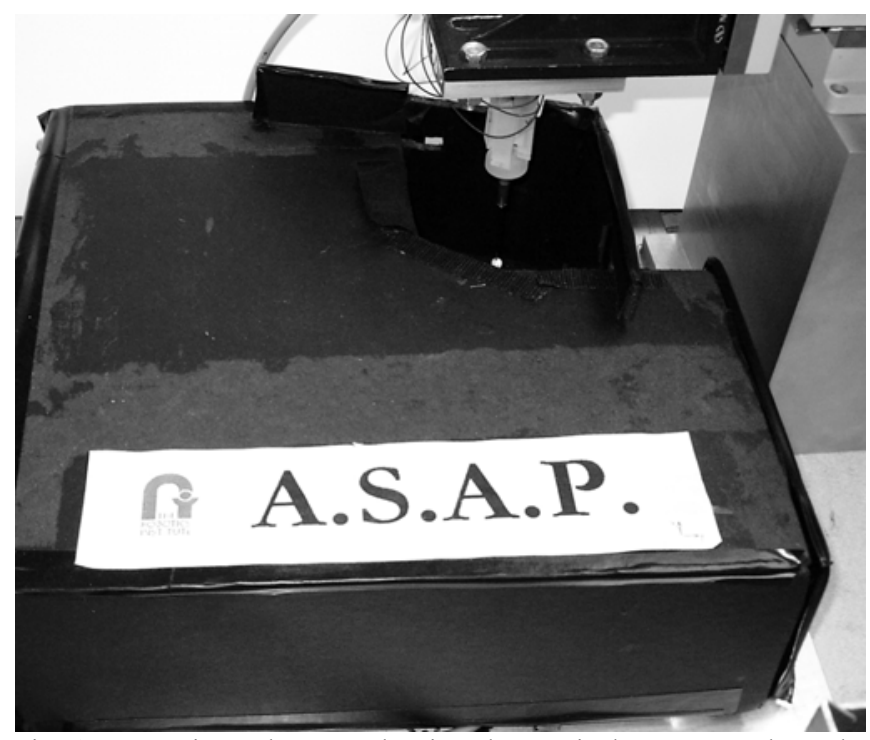

Fig. 2. Experimental setup, showing the manipulator mounted on the underside of a small platform near the top of the figure, and a small white marker ball at the tip. The 3-D motion of the tip is tracked via infrared illumination reflected by the ball.

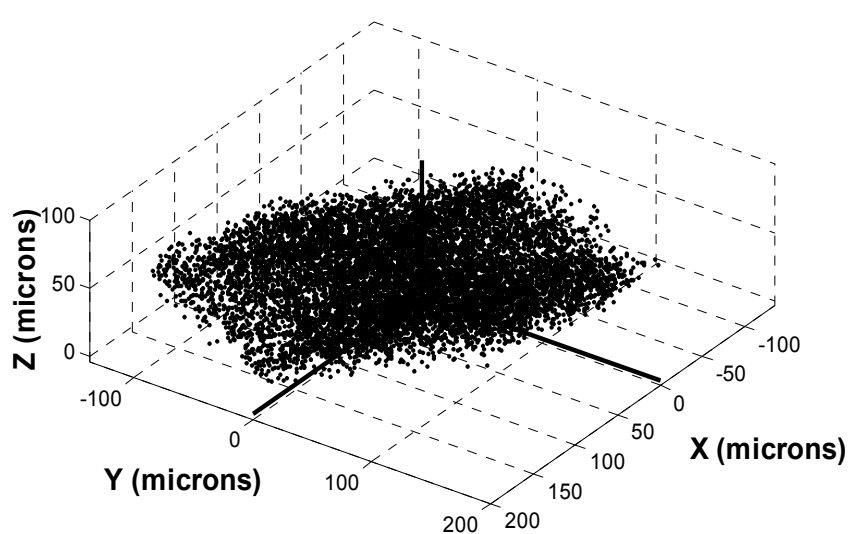

Fig 3. View of the manipulator workspace generated with 10000 randomly distributed points.

\section{RESULTS}

Table I shows the results of the training with different numbers of hidden nodes. The graphs in figure 4 show samples of the neural network results for the training data set. Figure 5 presents samples of results from the validation data set. The mean absolute error for the validation data is about $0.75 \mu \mathrm{m}$. The maximum error for the validation data set is $2.1 \mu \mathrm{m}$.

TABLE I

NeURAL NETWORK PERFORMANCE ON THE Training DATA SET

\begin{tabular}{ccc}
\hline \hline Hidden Nodes & MSE $\left(\mu \mathrm{m}^{2}\right)$ & MAE $(\mu \mathrm{m})$ \\
\hline 3 & 0.658 & 0.637 \\
4 & 0.643 & 0.628 \\
5 & 0.620 & 0.617 \\
6 & 0.615 & 0.614 \\
7 & 0.618 & 0.618 \\
8 & 0.601 & 0.644 \\
9 & 0.668 & 0.607 \\
$\mathrm{MSE}=$ mean squared error; $\mathrm{MAE}=$ mean of absolute error.
\end{tabular}

\section{DISCUSSION}

The data show the feasibility of the neural network for modeling the inverse kinematics of the manipulator. The fabrication errors of the stereolithographic process can be as large as several hundred microns. The accuracy target for the application is on the order of tens of microns [3], so these errors must be accounted for in the control of the manipulator. The effective dimensions of the fabricated flexure-based part are difficult to predict from the design, and difficult to measure from the fabricated part. The method presented here obviates these painstaking measurements of physical parameters. As such it may be an important tool for the development of the control system for the flexure-based micromanipulator. The method is rapid and therefore permits easy remodeling whenever changes in the design geometry take place. 


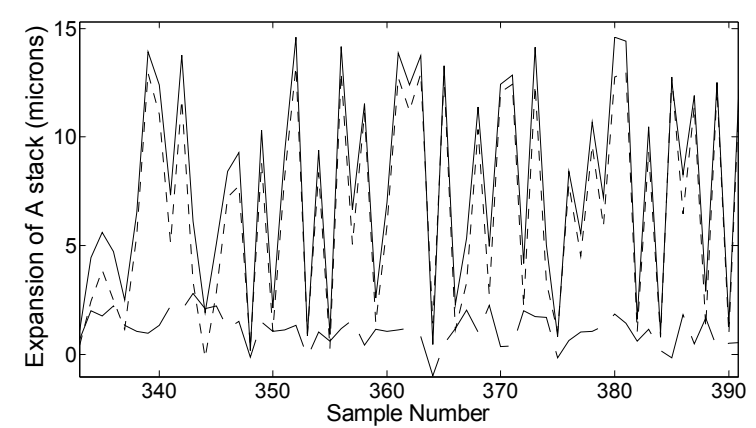

(a)

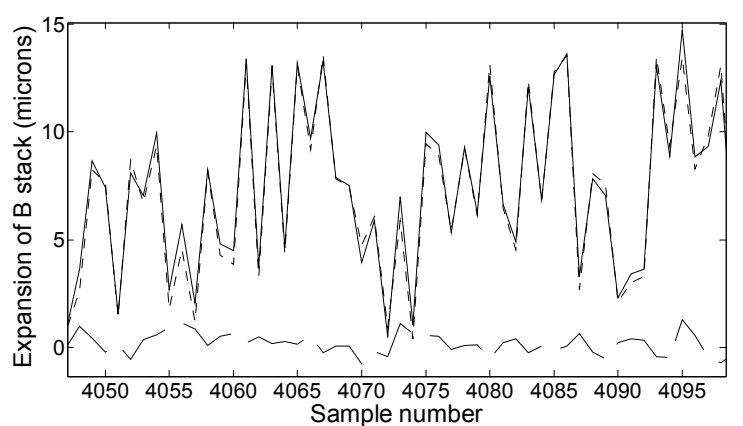

(b)

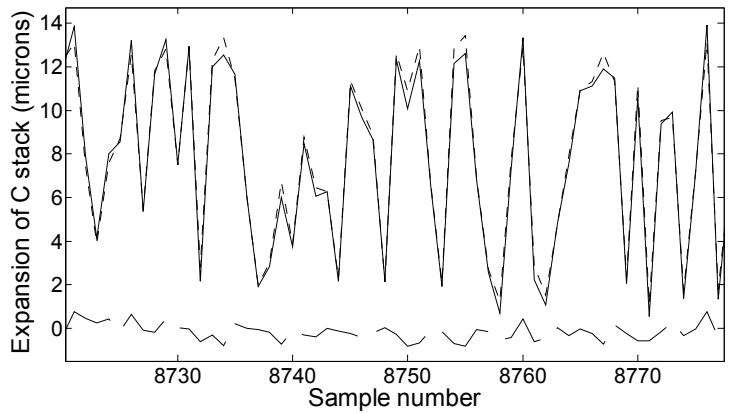

(c)

Fig 4. Sample results for the training data set for stacks A, B, and C, respectively. The solid line is the real input, the dotted line is the model response, and the dashed line is the error between the model and the real data.

\section{ACKNOWLEDGMENT}

J. G. A. thanks Juan Carlos Fraile Marinero of the University of Valladolid and C. N. Riviere for the opportunity provided as a visiting researcher at Carnegie Mellon University.

\section{REFERENCES}

[1] C. N. Riviere and P. K. Khosla, "Characteristics of hand motion of eye surgeons," Proc. 19th Annu. Conf. IEEE Eng. Med. Biol. Soc. Chicago, Oct. 30-Nov. 2, 1997, vol. 4, pp. 1690-1693.

[2] R. J. Elble and W. C. Koller, Tremor. Baltimore: Johns Hopkins, 1990.

[3] I. W. Hunter, T. D. Doukoglou, S. R. Lafontaine, P. G. Charette, L. A. Jones, M. A. Sagar, G. D. Mallinson, and P. J. Hunter, “A teleoperated microsurgical robot and associated virtual environment for eye surgery," Presence, vol. 2, pp. 265-280, 1993.

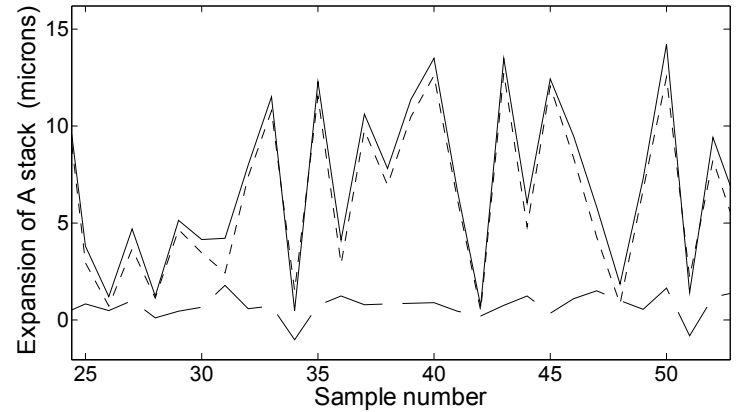

(a)

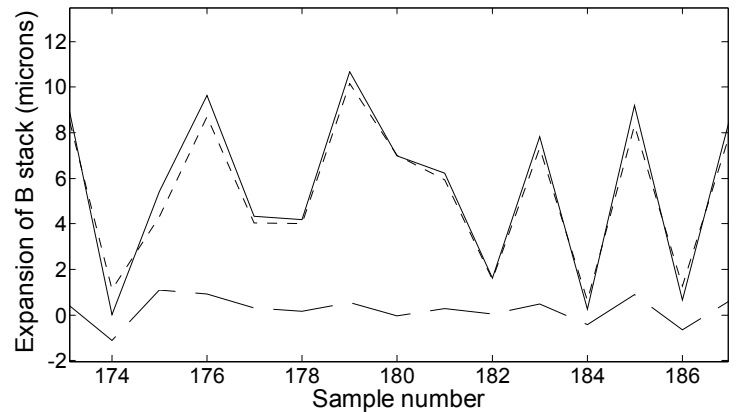

(b)

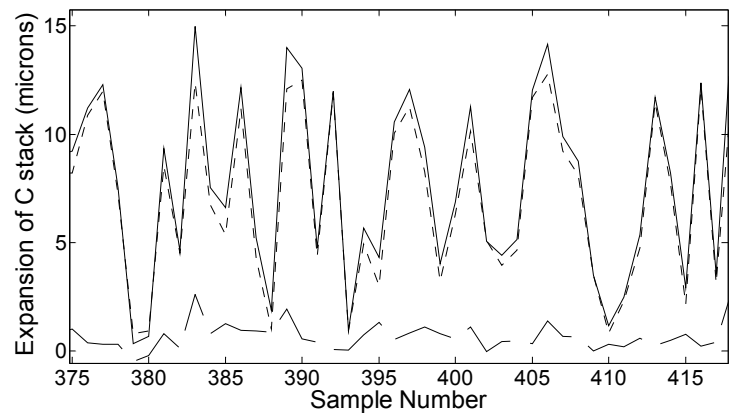

(c)

Fig 5. Sample results for the validation data set for stacks A, B, and C, respectively. The solid line is the real input, the dotted line is the mode response, and the dashed line is the error between the model and the real data.

[4] R. Taylor, P. Jensen, L. Whitcomb, A. Barnes, R. Kumar, D. Stoianovici, P. Gupta, Z. Wang, E. de Juan, Jr., and L. Kavoussi, "A steady-hand robotic system for microsurgical augmentation," Int. J. Robot. Res., vol. 18, pp. 1201-1210, 1999.

[5] C. N. Riviere, W. T. Ang, and P. K. Khosla, "Toward active tremor canceling in handheld microsurgical instruments," IEEE Trans. Rob. Autom., vol. 19, pp. 793-800, 2003.

[6] D. Y. Choi and C. N. Riviere, "Flexure-based manipulator for active handheld microsurgical instrument," 27th Annu. Intl. Conf. IEEE Eng. Med. Biol. Soc., Shanghai, China, Sept. 1-4, 2005, submitted.

[7] K. M Lee and D. K. Shah, "Kinematic analysis of a three-degrees offreedom in-parallel actuated manipulator," IEEE Trans. Robot. Autom., 4:354-360, 1988

[8] E. Oyama, N. Y. Chong, A. Agah, and T. Maeda, "Inverse kinematics learning by modular architecture neural networks with performance prediction networks," Proc. IEEE Intl. Conf. Robot. Autom., Seoul, Korea, 2001, vol. 1, pp. 1006-1012.

[9] B. M. Wilamowski, "Neural network architectures and learning," Proc. IEEE Intl. Conf. Industrial Technology, 2003, vol. 1, pp. TU1TU12. 